\title{
Thrombotic Complications of Nose and Toe Associated With Mycoplasma pneumoniae Infection
}

\author{
Hakan Tuncer ${ }^{\mathrm{a}}$, Sakir Omur Hincal ${ }^{\mathrm{a}}$, Betul Camª ${ }^{\mathrm{a}, \mathrm{b}}$
}

\begin{abstract}
Thrombosis is a very rare complication of Mycoplasma pneumoniae (M. pneumoniae) infection. We report a case of a woman who has thrombotic complications associated with mycoplasma infection in distal body parts. A 79-year-old female patient applied to the emergency department with complaints of the pain and discoloration at the nose tip and distal toes of the feet. Those symptoms have occurred in 10 days after the onset of the respiratory problems. The venous obstruction was diagnosed by venous Doppler ultrasound related to the distal part of the toes. The occurrence of the thrombosis associated with $M$. pneumoniae could be explained with some reaction of the inflammatory products in the blood circulation.
\end{abstract}

Keywords: Thrombosis; Mycoplasma pneumoniae; Inflammation

\section{Introduction}

Mycoplasma pneumoniae (M. pneumoniae) is known as a pathogen of the pulmonary system which causes extrapulmonary inflammatory problems in distant organ systems such as arthritis, hepatitis, pericarditis, hemolytic anemia, and central nervous system events $[1,2]$. Thrombosis is one of the extrapulmonary reactions of $M$. pneumoniae infection in rare clinical events. Some thrombus events have been reported in the cerebral arteries, intracardiac and splenic arteries, previously [3-8]. Pulmonary embolism and the thrombosis of pediatric femoral artery have also been reported in children related to $M$. pneumoniae infection $[9,10]$. Clinical symptoms might be explained by coagulation abnormalities, thrombocytosis, thrombocytopenia or some antibody reactions [11-14].

A case report of a thrombus event of M. pneumoniae infection and the clinical course of recovery after the treatment with antibiotherapy and anticoagulant treatment in a 79-year-old

Manuscript submitted July 23, 2019, accepted August 6, 2019

aDepartment of Emergency Medicine, Bagcilar Training and Research Hospital, Istanbul, Turkey

${ }^{b}$ Corresponding Author: Betul Cam, Department of Emergency Medicine, Bagcilar Training and Research Hospital, Istanbul 34214, Turkey.

Email:mbetulcam@gmail.com woman who complained of pain and discoloration on the nose and distal part of the toes was presented.

\section{Case Report}

A 79-year-old woman applied to the hospital with the complaints of severe pain and discoloration on her nose tip and the distal parts of the toes following persistent fever and cough about 10 days. She had rale sounds on the lungs. Bilateral common consolidation was found in the lungs on thorax computed tomography (CT). According to the history of the patient, the discoloration was emerged after about 10 days by the beginning of the fever and cough. The venous obstruction was observed with venous Doppler ultrasonography on the plantar digital area of the feet (Figs. 1, 2). The patient was hospitalized with anticoagulant treatment and antibiotherapy. After antibiotherapy (moxifloxacin) and anticoagulant (enoxaparin) treatment, the nose and distal toe area were healed. Three months after the treatment, the patient was observed in a healthy status and did not have a recurrent thrombosis or thromboembolism complication ascertained by the telephone interview with her family.

\section{Discussion}

M. pneumoniae is a common cause of atypical pneumonia often seen in youths and rarely in adults [1]. M. pneumoniae

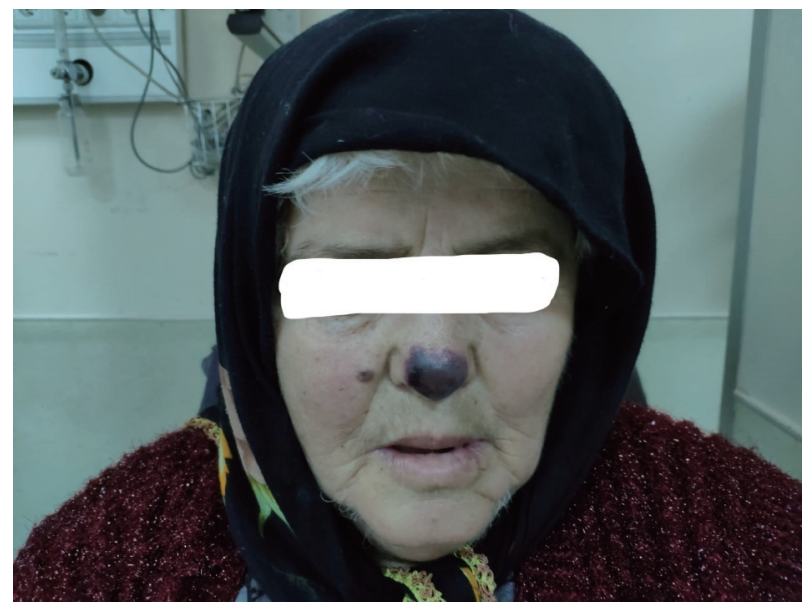

Figure 1. Thrombosis on the tip of the nose. 


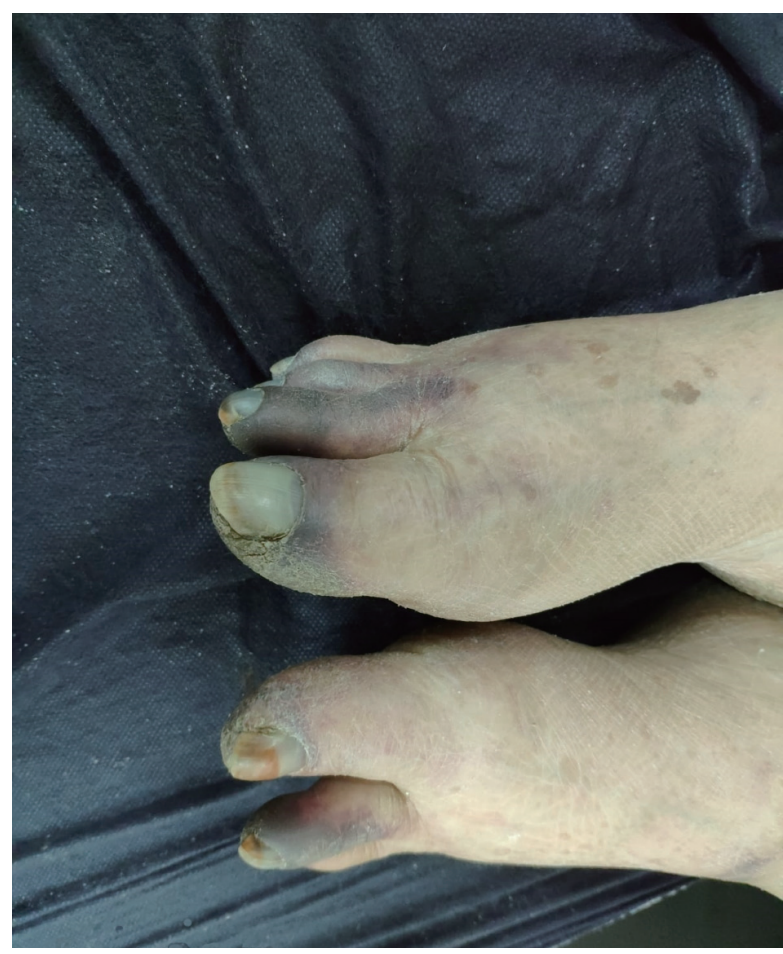

Figure 2. Thrombosis in the toe area.

pneumonia occurs typically in a mild condition and is characterized by a persistent dry cough, and sometimes self-limiting pneumonia cured with no medication, fulminant cases with severe complications such as respiratory failure, hypoxia, and extrapulmonary manifestations $[1,2]$. Some cases have been reported that following $M$. pneumoniae infection, acute cerebral infarction, intracardiac or splenic thrombus was commonly detected in children [3-8]. It has been considered that the circulation problems caused by abnormal coagulation or thrombosis could be responsible for the extrapulmonary complications of the infection $[9,10]$. However, the mechanism of the coagulation problems and thrombosis has not been fully understood. Some authors concluded that the release of cytokines with mycoplasma infection produced the vascular wall inflammation, leading to local vasculitis and thrombosis. Also, some studies have shown that activation of the complement system caused by mycoplasma infection activates the coagulation system [11-13]. The mechanism of abnormal coagulation and the promotion of thrombosis after $M$. pneumoniae infection could be explained by the activation of exogenous and endogenous coagulation systems by multiple pathways which provokes the imbalance in coagulation and anticoagulation factors. Some inflammatory factors, such as tumor necrosis factor- $\alpha$ and interleukin, might accelerate the coagulation. In the process of inflammatory reactions, the synthesis of some anticoagulation factors such as antithrombin III (AT-III) and protein C is affected, which results in the promotion of coagulation. It has been reported that AT-III levels and protein $\mathrm{C}$ activity are significantly different in $M$. pneumoniae-infected children compared to the healthy children, and after anti-mycoplasma treatment, protein $\mathrm{C}$ activity returns to normal $[9,10]$.
Thrombus caused by M. pneumoniae infection has been successfully treated with oral or parenteral anticoagulant therapy such as intravenous heparin, intra-subcutaneous injection of low molecular weight heparin, and oral administration of warfarin, in previously reported cases [6-10]. Local vasculitis and thrombotic vascular occlusion disappeared within a few weeks to months following the treatment of $M$. pneumoniae infection.

In conclusion, M. pneumoniae infection may cause thrombosis formation and transient production of the antiphospholipid antibodies which may involve its mechanism. Thrombolytic therapies should be taken into account as soon as possible when the appearance of $M$. pneumoniae pneumonia clinical symptoms has been detected.

\section{Acknowledgments}

The authors have no acknowledgments to state.

\section{Financial Disclosure}

This was an unfunded evaluation.

\section{Conflict of Interest}

The authors have no conflict of interest to disclose.

\section{Informed Consent}

All participants enrolled in the study provided written informed consent.

\section{Author Contributions}

All of the authors have contributed sufficiently to the project through drafting and revising the manuscript to be included as authors.

\section{References}

1. Bajantri B, Venkatram S, Diaz-Fuentes G. Mycoplasma pneumoniae: A Potentially Severe Infection. J Clin Med Res. 2018;10(7):535-544.

2. Narita M. Pathogenesis of extrapulmonary manifestations of Mycoplasma pneumoniae infection with special reference to pneumonia. J Infect Chemother. 2010;16(3):162169.

3. Nagashima M, Higaki T, Satoh H, Nakano T. Cardiac thrombus associated with Mycoplasma pneumoniae infection. Interact Cardiovasc Thorac Surg. 2010;11(6):849851.

4. Kang B, Kim DH, Hong YJ, Son BK, Lim MK, Choe 
YH, Kwon YS. Complete occlusion of the right middle cerebral artery associated with Mycoplasma pneumoniae pneumonia. Korean J Pediatr. 2016;59(3):149-152.

5. Kim GH, Seo WH, Je BK, Eun SH. Mycoplasma pneumoniae associated stroke in a 3-year-old girl. Korean J Pediatr. 2013;56(9):411-415.

6. Antachopoulos C, Liakopoulou T, Palamidou F, Papathanassiou D, Youroukos S. Posterior cerebral artery occlusion associated with Mycoplasma pneumoniae infection. J Child Neurol. 2002;17(1):55-57.

7. Bakshi M, Khemani C, Vishwanathan V, Anand RK, Khubchandani RP. Mycoplasma pneumonia with antiphospholipid antibodies and a cardiac thrombus. Lupus. 2006;15(2):105-106.

8. Witmer CM, Steenhoff AP, Shah SS, Raffini LJ. Mycoplasma pneumoniae, splenic infarct, and transient antiphospholipid antibodies: a new association? Pediatrics. 2007;119(1):e292-295.

9. Brown SM, Padley S, Bush A, Cummins D, Davidson S, Buchdahl R. Mycoplasma pneumonia and pulmonary embolism in a child due to acquired prothrombotic fac- tors. Pediatr Pulmonol. 2008;43(2):200-202.

10. Graw-Panzer KD, Verma S, Rao S, Miller ST, Lee H. Venous thrombosis and pulmonary embolism in a child with pneumonia due to Mycoplasma pneumoniae. J Natl Med Assoc. 2009;101(9):956-958.

11. Cunha BA, Pherez FM. Mycoplasma pneumoniae community-acquired pneumonia (CAP) in the elderly: Diagnostic significance of acute thrombocytosis. Heart Lung. 2009;38(5):444-449.

12. Okoli K, Gupta A, Irani F, Kasmani R. Immune thrombocytopenia associated with Mycoplasma pneumoniae infection: a case report and review of literature. Blood Coagul Fibrinolysis. 2009;20(7):595-598.

13. Snowden N, Wilson PB, Longson M, Pumphrey RS. Antiphospholipid antibodies and Mycoplasma pneumoniae infection. Postgrad Med J. 1990;66(775):356-362.

14. Padovan CS, Pfister HW, Bense S, Fingerle V, Abele-Horn M. Detection of Mycoplasma pneumoniae DNA in cerebrospinal fluid of a patient with M. pneumoniae infection"associated" stroke. Clin Infect Dis. 2001;33(10):E119121. 